\title{
Numerical Solution of Optimal Control Problems for Parabolic Systems
}

\author{
Peter Benner ${ }^{1}$, Sabine Görner ${ }^{1}$, and Jens Saak ${ }^{1}$ \\ Professur Mathematik in Industrie und Technik, \\ Fakultät für Mathematik, \\ Technische Universität Chemnitz, \\ 09107 Chemnitz, \\ Germany. \\ \{benner, sabine.goerner, jens.saak\}@mathematik.tu-chemnitz.de
}

Summary. We discuss the numerical solution of optimal control problems for instationary convection-diffusion and diffusion-reaction equations. Instead of viewing this problem as a large-scale unconstrained optimization problem after complete discretization of the corresponding optimality system, we formulate the problem as abstract linear-quadratic regulator (LQR) problem. Using recently developed efficient solvers for large-scale algebraic Riccati equations, we show how to numerically solve the optimal control problem at a cost proportional to solving the corresponding forward problem. We discuss two different optimization goals: one can be seen as stabilization of the plant model, the second one is of tracking type, i.e., a given (optimal) solution trajectory is to be attained. The efficiency of our approach is demonstrated for a model problem related to an optimal cooling process. Moreover, we discuss how the LQR approach can be applied to nonlinear problems.

\section{Introduction}

We consider nonlinear parabolic diffusion-convection and diffusion-reaction systems of the form

$$
\frac{\partial \mathbf{x}}{\partial t}+\nabla \cdot(\mathbf{C}(\mathbf{x})-\mathbf{K}(\nabla \mathbf{x}))-\mathbf{Q}(\mathbf{x})=\mathcal{B} \mathbf{u}(t), \quad t \in\left[0, T_{f}\right],
$$

in $\Omega \in \mathbb{R}^{d}, d=1,2,3$, with appropriate initial and boundary conditions. Here, $\mathbf{C}$ is the convective part, $\mathbf{K}$ the diffusive part and $\mathbf{Q}$ is an uncontrolled source term. The state of the system is denoted by $\mathbf{x}$ and the control by $\mathbf{u}$. A control problem is defined as

$$
\min _{\mathbf{u}} J(\mathbf{x}, \mathbf{u}) \quad \text { subject to }(1)
$$

where $J(\mathbf{x}, \mathbf{u})$ is a performance index which will be introduced later. 
There are two possibilities for the appearance of the control. If the control occurs in the boundary condition, we call this problem a boundary control problem. It is called distributed control problem if the control acts in $\Omega$ or a sub-domain $\Omega_{u} \subset \Omega$. The control problem as in (1) is well-suited to describe a distributed control problem while boundary control will require the specification of the boundary conditions as, for instance, given below.

The major part of this article deals with the linear version of (1),

$$
\frac{\partial \mathbf{x}}{\partial t}-\nabla \cdot(a(\xi) \nabla \mathbf{x})+d(\xi) \nabla \mathbf{x}+r(\xi) \mathbf{x}=\mathbf{B}_{V}(\xi) u(t), \quad \xi \in \Omega, t>0,
$$

with initial and boundary conditions

$$
\begin{aligned}
\alpha(\xi) \frac{\partial \mathbf{x}(t)}{\partial n}+\gamma(\xi) \mathbf{x}(t) & =\mathbf{B}_{R} u(t), \quad \xi \in \partial \Omega, \\
\mathbf{x}(0)(\xi) & =\mathbf{x}_{0}(\xi), \quad \xi \in \Omega,
\end{aligned}
$$

for sufficiently smooth parameters $a, d, r, \alpha, \gamma, \mathbf{x}_{0}$. We assume that either $\mathbf{B}_{V}=0$ (boundary control system) or $\mathbf{B}_{R}=0$ (distributed control system). In addition, we include in our problem an output equation of the form

$$
\mathbf{y}=\mathbf{C x}, \quad t \geq 0,
$$

taking into account that in practice, often not the whole state $\mathbf{x}$ is available for measurements. Here, $\mathbf{C}$ is a linear operator which often is a restriction operator.

To solve optimal control problems (2) with a linear system (3) we interpret it as a linear quadratic regulator (LQR) problem. The theory behind the LQR ansatz has already been studied in detail, e.g., in $[32,33,34,35,12,2]$, to name only a few.

Nonlinear control problems are still undergoing extensive research. We will apply model predictive control (MPC) here, i.e., we solve linearized problems on small time frames using a linear-quadratic Gaussian (LQG) design. This idea is presented by Ito and Kunisch in [29]. We will briefly sketch the main ideas of this approach at the end of this article.

There exists a rich variety of other approaches to solve linear and nonlinear optimal control problems for partial differential equations. We can only refer to a selection of ideas, see e.g. [41, 11, 23, 35, 25, 26].

This article is divided into four parts. In the remainder of this section we will give a short introduction to linear control problems and we present the model problem used in this article. Theoretical results which justify the numerical implementation of the LQR problem will be pointed out in Section 2. The third section deals with computational methods for the model problem. There we go into algorithmic and implementation details and present some numerical results. Finally we give an insight into a method for nonlinear parabolic systems in Section 4. 


\subsection{Linear Problems}

In this section we will formulate the linear quadratic regulator (LQR) problem. We assume that $\mathcal{X}, \mathcal{Y}, \mathcal{U}$ are separable Hilbert spaces where $\mathcal{X}$ is called the state space, $\mathcal{Y}$ the observation space and $\mathcal{U}$ the control space.

Furthermore the linear operators

$$
\mathbf{A}: \operatorname{dom}(\mathbf{A}) \subset \mathcal{X} \rightarrow \mathcal{X}, \quad \mathbf{B}: \mathcal{U} \rightarrow \mathcal{X}, \quad \mathbf{C}: \mathcal{X} \rightarrow \mathcal{Y}
$$

are given. Such an abstract system can now be understood as a Cauchy problem for a linear evolution equation of the form

$$
\dot{\mathbf{x}}=\mathbf{A} \mathbf{x}+\mathbf{B u}, \quad \mathbf{x}(0)=\mathbf{x}_{0} \in \mathcal{X} .
$$

Since in many applications the state $\mathbf{x}$ of a system can not be observed completely we consider the observation equation

$$
\mathbf{y}=\mathbf{C x}
$$

which describes the map between the states and the outputs of the system.

The abstract LQR problem is now given as the minimization problem

$$
\min _{\mathbf{u} \in L^{2}\left(0, T_{f} ; \mathcal{U}\right)} \frac{1}{2} \int_{0}^{T_{f}}\langle\mathbf{y}, \mathbf{Q y}\rangle_{\mathcal{Y}}+\langle\mathbf{u}, \mathbf{R u}\rangle_{\mathcal{U}} d t
$$

with self-adjoint, positive definite, linear, bounded operators $\mathbf{Q}$ and $\mathbf{R}$ on $\mathcal{Y}$ and $\mathcal{U}$, respectively. Recall that in case (4) is an ordinary differential equation with $\mathcal{X}=\mathbb{R}^{n}, \mathcal{Y}=\mathbb{R}^{p}$ and $\mathcal{U}=\mathbb{R}^{m}$ are equipped with the standard scalar product, then we obtain an LQR problem for a finite-dimensional system [40]. For partial differential equations we have to choose the function spaces $\mathcal{X}, \mathcal{Y}, \mathcal{U}$ appropriately and we get an LQR system for an infinite-dimensional system $[17,18]$.

Many optimal control problems for instationary linear partial differential equations can be described using the abstract LQR problem above. Additionally, many control, stabilization and parameter identification problems can be reduced to the LQR problem, see [3, 18, 32, 33, 34].

\section{The Infinite Time Case}

In the infinite time case we assume that $T_{f}=\infty$ so that we have to solve the minimization problem

$$
\min _{\mathbf{u} \in L^{2}(0, \infty ; \mathcal{U})} \frac{1}{2} \int_{0}^{\infty}\langle\mathbf{y}, \mathbf{Q y}\rangle_{\mathcal{Y}}+\langle\mathbf{u}, \mathbf{R u}\rangle_{\mathcal{U}} d t
$$

subject to (4).

If the standard assumptions that 
- $\mathbf{A}$ is the infinitesimal generator of a $\mathcal{C}^{0}$-semigroup $\mathbf{T}(t)$,

- $\mathbf{B}, \mathbf{C}$ are linear bounded operators and

- for every initial value there exists an admissible control $\mathbf{u} \in L^{2}(0, \infty ; \mathcal{U})$

hold then the solution of the abstract LQR problem can be obtained analogously to the finite-dimensional case (see [44, 17, 21]). We then have to consider the algebraic operator Riccati equation

$$
0=\Re(\mathbf{P})=\mathbf{C}^{*} \mathbf{Q} \mathbf{C}+\mathbf{A}^{*} \mathbf{P}+\mathbf{P A}-\mathbf{P B R}^{-1} \mathbf{B}^{*} \mathbf{P},
$$

where the linear operator $\mathbf{P}$ will be the solution of (8) if $\mathbf{P}: \operatorname{dom} \mathbf{A} \rightarrow \operatorname{dom} \mathbf{A}^{*}$ and $\langle\hat{\mathbf{x}}, \Re(\mathbf{P}) \mathbf{x}\rangle=0$ for all $\mathbf{x}, \hat{\mathbf{x}} \in \operatorname{dom}(\mathbf{A})$. The optimal control is then given as the feedback control

$$
\mathbf{u}_{*}(t)=-\mathbf{R}^{-1} \mathbf{B}^{*} \mathbf{P}_{\infty} \mathbf{x}_{*}(t),
$$

which has the form of a regulator or closed-loop control. Here, $\mathbf{P}_{\infty}$ is the minimal nonnegative self-adjoint solution of $(8), \mathbf{x}_{*}(t)=\mathbf{S}(t) \mathbf{x}_{0}(t)$, and $\mathbf{S}(t)$ is the $\mathcal{C}^{0}$-semigroup generated by $\mathbf{A}-\mathbf{B} \mathbf{R}^{-1} \mathbf{B}^{*} \mathbf{P}_{\infty}$. Using further standard assumptions it can be shown, see e.g. [12], that $\mathbf{P}_{\infty}$ is the unique nonnegative solution of (8). Most of the required conditions, particularly the restrictive assumption that $\mathbf{B}$ is bounded, can be weakened [32, 33].

\section{The Finite Time Case}

The finite time case arises if $T_{f}<\infty$ in (6). Then the numerical solution is more complicated since we have to solve the operator differential Riccati equation

$$
\dot{\mathbf{P}}(t)=-\left(\mathbf{C}^{*} \mathbf{Q} \mathbf{C}+\mathbf{A}^{*} \mathbf{P}(t)+\mathbf{P}(t) \mathbf{A}-\mathbf{P}(t) \mathbf{B R}^{-1} \mathbf{B}^{*} \mathbf{P}(t)\right) .
$$

The optimal control is obtained as

$$
\mathbf{u}_{*}(t)=-\mathbf{R}^{-1} \mathbf{B}^{*} \mathbf{P}_{*}(t) \mathbf{x}_{*}(t),
$$

where $\mathbf{P}_{*}(t)$ is the unique solution of (10) in complete analogy to the infinite time case in (9).

\subsection{Discretization}

For the discretization of an optimal control problem we can follow different strategies. In the literature the following two alternatives are often used:

- "Optimize-then-discretize"

That is, we compute the optimal control with methods of optimization first and discretize afterwards.

- "Discretize-then-optimize"

Here, we discretize at first and optimize the discrete problem. 

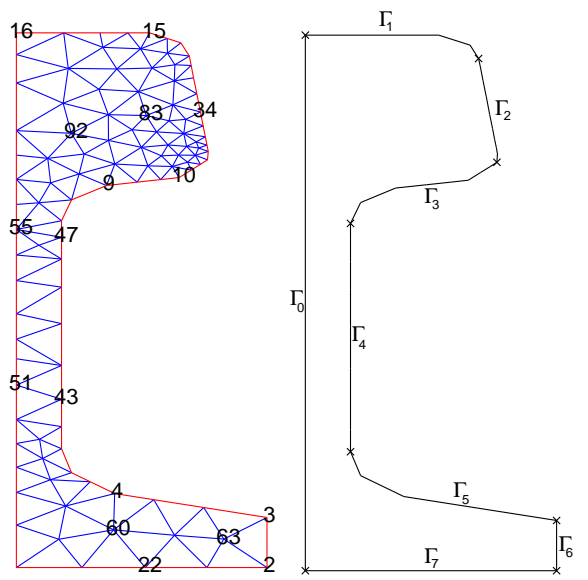

Fig. 1. Initial mesh with observation nodes (left) and partitioning of the boundary (right).

The literature provides a large amount of approaches which are based on non-smooth Newton's methods or sequential quadratic programming (SQP) methods, see, e.g., [13, 25, 26].

In contrast to the strategies above we want to examine the strategy "semidiscretize-optimize-semidiscretize". If we semidiscretize in space first, for instance by using a Galerkin ansatz with finite element ansatz functions, we obtain a linear finite-dimensional system. The structure and solution of the resulting system are analogous to those of the infinite dimensional system.

Thus, we can formulate the following general strategy for solving LQR problems, where, of course, the applicability of the described Riccati approach has to be tested for every situation, in particular the conditions on the regularity of the boundary and the solution play a decisive role.

1. Find a spatial discretization for the partial differential equation using a Galerkin projection of $\mathcal{X}$ on a finite-dimensional subspace $\mathcal{X}^{N}$ with matrix representations $A^{N}, B^{N}, C^{N}, Q^{N}$ of the corresponding linear operators $\mathbf{A}, \mathbf{B}, \mathbf{C}, \mathbf{Q}$.

2. Solve the finite-dimensional LQR problem.

3. Apply the finite-dimensional feedback law to the infinite-dimensional system.

4. If necessary, refine the discretization.

\subsection{The Model Problem}

The control of the cooling process for a rail profile in a rolling mill serves as a benchmark problem for our approach. The model has been discussed in detail in the literature in the context of optimization by Tröltzsch and others (see 
$[42,19,31]$ and references therein). First results concerning the LQR design for this problem can be found in $[38,8,10,9]$.

As in $[42,19,31,20]$ the steel profile is assumed to stretch infinitely into the $z$-direction. This admits the assumption of a stationary heat distribution in $z$-direction. That means we can restrict ourselves to a 2 -dimensional heat diffusion process on cross-sections of the profile $\Omega \subset \mathbb{R}^{2}$ as shown in Figure 1 . Measurements for defining the geometry of the cross-section are taken from [42]. As one can see in Figure 1 the domain exploits the symmetry of the profile introducing an artificial boundary $\Gamma_{0}$ on the symmetry axis.

We will concentrate on the linearized version of the state equation introduced in $[42,19,31]$. The linearization is derived by taking means of the material parameters $\rho, \lambda$ and $c$. This is admissible as long as we work in temperature regimes above approximately $700-800^{\circ} \mathrm{C}$ (depending on the kind of steel used) where changes of $\rho, \lambda$ and $c$ are small and we do not have multiple phases and phase transitions in the material. We partition the non-artificial boundary into 7 parts, each of them located between two neighboring corners of the domain (see Figure 1 for details). The control $u$ is assumed to be constant with respect to the spatial variable $\xi$ on each part $\Gamma_{i}$ of the boundary. Thus we obtain the following model:

$$
\begin{aligned}
c \rho \frac{\partial x(\xi, t)}{\partial t} & =\nabla \cdot(\lambda \nabla x(\xi, t)) & & \text { in } \Omega \times(0, T), \\
-\lambda \frac{\partial x(\xi, t)}{\partial n} & =g_{i}(t, x, u) & & \text { on } \Gamma_{i} \text { for } i=0, \ldots, 7, \\
x(\xi, 0) & =x_{0}(\xi) & & \text { in } \Omega .
\end{aligned}
$$

We now have to describe the heat transfer across the surface of the material, i.e. the boundary conditions. The boundary condition according to Newton's cooling law is given as

$$
-\frac{\partial x(\xi, t)}{\partial n}=\frac{\kappa_{k}}{\lambda}\left(x(\xi, t)-x_{e x t, k}(t)\right) .
$$

Note that $x_{e x t, k}(t)$ is assumed to be constant on $\Gamma_{k}$ and therefore does not depend explicitly on $\xi$. For a more detailed derivation of this condition see [9].

Here, we will take the external temperature as the control. The mathematical advantage of this choice is that the multiplication of control and state which would lead to a bilinear control system in case of the heat transfer coefficient as control is avoided.

\section{Theoretical Results}

\subsection{Approximation of Abstract Cauchy Problems}

The theoretical fundament for our approach was set by Gibson [21]. The ideas and proofs used for the boundary control problem considered here closely follow the extension of Gibson's method proposed by Banks and Kunisch [4] 
for distributed control systems arising from parabolic equations. Similar approaches can be found in $[9,33]$. Common to all those approaches is to formulate the control system for a parabolic system as an abstract Cauchy problem in an appropriate Hilbert space setting. For numerical approaches this Hilbert space $\mathcal{X}$ is approximated by a sequence of finite-dimensional spaces $\left(\mathcal{X}^{N}\right)_{N \in \mathbb{N}}$, e.g., by spatial finite element approximations, leading to large sparse systems of ordinary differential equations in $\mathbb{R}^{n}$. Following the theory in [4] those approximations do not even have to be subspaces of the Hilbert space of solutions.

Before stating the main theoretical result we will first collect some approximation prerequisites we will need for the theorem. We call them (BK1) and (BK2) for they were already formulated in [4] (and called $\mathrm{H} 1$ and $\mathrm{H} 2$ there). In the following $\Pi^{N}$ is the canonical projection operator mapping from the infinite-dimensional space $\mathcal{X}$ to its finite-dimensional approximation $\mathcal{X}^{N}$. The first and natural prerequisite is:

For each $N$ and $x_{0} \in \mathcal{X}^{N}$ there exists an admissible control $u^{N} \in L^{2}(0, \infty ; \mathcal{U})$ and any admissible control drives the states to 0 asymptotically.

Additionally one needs the following properties for the approximation as $N \rightarrow \infty$. Assume that for each $N, A^{N}$ is the infinitesimal generator of a $\mathcal{C}^{0}$-semigroup $T^{N}(t)$, then we require:

(i) For all $\varphi \in \mathcal{X}$ we have uniform convergence $T^{N}(t) \Pi^{N} \varphi \rightarrow \mathbf{T}(t) \varphi$ on any bounded subinterval of $[0, \infty)$.

(ii) For all $\phi \in \mathcal{X}$ we have uniform convergence $T^{N}(t)^{*} \Pi^{N} \phi \rightarrow \mathbf{T}(t)^{*} \phi$ on any bounded subinterval of $[0, \infty)$.

(iii) For all $v \in \mathcal{U}$ we have $B^{N} v \rightarrow \mathbf{B} v$ and for all $\varphi \in \mathcal{X}$ we have $B^{N^{*}} \varphi \rightarrow \mathbf{B}^{*} \varphi$.

(iv) For all $\varphi \in \mathcal{X}$ we have $Q^{N} \Pi^{N} \varphi \rightarrow \mathbf{Q} \varphi$.

With these we can now formulate the main result.

Theorem 1 (Convergence of the finite-dimensional approximations). Let (BK1) and (BK2) hold. Moreover, assume $\mathbf{R}>0, \mathbf{Q} \geq 0$ and $Q^{N} \geq 0$. Further, let $P^{N}$ be the solutions of the AREs for the finite-dimensional systems and let the minimal nonnegative self-adjoint solution $\mathbf{P}$ of (8) for (4), (5) and (7) exist. Moreover, let $\mathbf{S}(t)$ and $S^{N}(t)$ be the operator semigroups generated by $\mathbf{A}-\mathbf{B R}^{-1} \mathbf{B}^{*} \mathbf{P}$ on $\mathcal{X}$ and $A^{N}-B^{N} \mathbf{R}^{-1} B^{N^{*}} P^{N}$ on $\mathcal{X}^{N}$, respectively, with $\|\mathbf{S}(t) \varphi\| \rightarrow 0$ as $t \rightarrow \infty$ for all $\varphi \in \mathcal{X}$.

If there exist positive constants $M_{1}, M_{2}$ and $\omega$ independent of $N$ and $t$, such that 


$$
\begin{aligned}
\left\|S^{N}(t)\right\|_{\mathcal{X}^{N}} & \leq M_{1} e^{-\omega t}, \\
\left\|P^{N}\right\|_{\mathcal{X}^{N}} & \leq M_{2},
\end{aligned}
$$

then

$$
\begin{array}{rlrl}
P^{N} \Pi^{N} \varphi & \rightarrow \mathbf{P} \varphi & & \text { for all } \varphi \in \mathcal{X}, \\
S^{N}(t) \Pi^{N} \varphi & \rightarrow \mathbf{S}(t) \varphi & \text { for all } \varphi \in \mathcal{X},
\end{array}
$$

converge uniformly in $t$ on bounded subintervals of $[0, \infty)$ as $N \rightarrow \infty$ and

$$
\|\mathbf{S}(t)\| \leq M_{1} e^{-\omega t} \text { for } t \geq 0 .
$$

Theorem 1 gives the theoretical justification for the numerical method used for the linear problems described in this paper. It shows that the finitedimensional closed-loop system obtained from optimizing the semidiscretized control problem indeed converges to the infinite-dimensional closed-loop system. Deriving a similar result for the nonlinear problem is an open problem.

The proof of Theorem 1 is given in [9]. It very closely follows that of [4, Theorem 2.2]. The only difference is the definition of the sesquilinear form on which the mechanism of the proof is based. It has an additional term in the boundary control case discussed here, but one can check that this term does not destroy the required properties of the sesquilinear form.

\subsection{Tracking Control}

In contrast to stabilization problems, where one searches for a stabilizing feedback $K$ (i.e. a feedback such that the closed loop operator $A-B K$ is stable), we are searching for a feedback which drives the state to a given reference trajectory asymptotically. Thus the tracking problem is in fact a stabilization problem for the deviation of the current state from the desired state. We will show in this section, that for linear operators $A$ and $B$ tracking can easily be incorporated into an existing solver for the stabilization problem with only a small computational overhead.

A common trick (see, e.g., [22]) to handle inhomogeneities in system theory for ODEs is the following. Given

$$
\dot{x}=A x+B u+f,
$$

let $\hat{x}$ be a solution of the uncontrolled system $\dot{x}=A x+f$, such that $f=\dot{\hat{x}}-A \hat{x}$. Then

$$
\dot{x}-\dot{\hat{x}}=A x+B u-A \hat{x}
$$

from which we get a homogenous linear system

$$
\dot{z}=A z+B u \quad \text { where } \quad z=x-\hat{x} .
$$

We want to apply this to the abstract Cauchy problem. Assume ( $\tilde{\mathbf{x}}, \tilde{\mathbf{u}})$ is a reference pair solving

$$
\dot{\tilde{\mathbf{x}}}=\mathbf{A} \tilde{\mathbf{x}}+\mathbf{B} \tilde{\mathbf{u}} \text {. }
$$


We rewrite the tracking type control system as a stabilization problem for the difference $\mathbf{z}=\mathbf{x}-\tilde{\mathbf{x}}$ as follows:

$$
\dot{\mathbf{z}}=\mathbf{A z}+\mathbf{B v} \quad \stackrel{\mathbf{v}=-K \mathbf{z}}{\Leftrightarrow} \dot{\mathbf{x}}=\mathbf{A x}-\mathbf{B K} \mathbf{x}+\dot{\tilde{\mathbf{x}}}-\mathbf{A} \tilde{\mathbf{x}}+\mathbf{B K} \tilde{\mathbf{x}} .
$$

So the only difference between the tracking type and stabilization problems is the known inhomogeneity $\mathbf{f}:=\dot{\tilde{\mathbf{x}}}-(\mathbf{A}-\mathbf{B K}) \tilde{\mathbf{x}}$. Note that the operators do not change at all. That means we have to solve the same Riccati equation (8) in both cases, thus one only has to add the inhomogeneity $\mathbf{f}$ (which can be computed once and in advance directly after the feedback operator is obtained) to the solver for the closed loop system in the tracking type case provided that in the cost function (7) $\mathbf{y}=\mathbf{C x}$ has been replaced by $\mathbf{C}(\mathbf{x}-\tilde{\mathbf{x}})$.

\section{Computational Methods and Results}

In this section we will discuss the computational methods used to achieve an efficient implementation for the numerical solution of the model problem. In Subsection 3.1 we will first explain the algorithms used to solve the problem. There we will especially review the algorithm employed to solve the large sparse Riccati equation. We will focus on the case of infinite final time, where we have to deal with an algebraic Riccati equation (ARE), but we will also sketch the method used for a differential Riccati equation (DRE) in the finite final time case. After that we will briefly explain the concrete implementation in Subsection 3.2 and give an overview of the problems which may be solved with our implementation at the current stage. In the closing subsection we will present selected numerical results of our computations for tracking type control systems. Numerical experiments for the stabilization problem have already been published in $[38,8,9,10]$.

\subsection{Algorithmic Details}

The approach we present here admits two different implementations which can be seen as implementations of the well known horizontal and vertical methods of lines from numerical methods for partial differential equations. In the case of the vertical method of lines we use a finite element semidiscretization in space to set up the approximate finite-dimensional problems. This approximation is then used to formulate an LQR system for an ordinary differential equation. The LQR system for this ordinary differential equation is then solved by computing the feedback, retrieving the closed loop system and applying an ODE solver to the closed loop system. The case of the horizontal method of lines is very similar to the algorithm used when solving the PDE forward problem. We only have to introduce a step computing the feedback operator and a step which updates the boundary conditions according to this operator for the boundary control system. 
So in both cases we need to compute the feedback operator for the approximate finite-dimensional systems. As we use finite element approximations here we have to deal with matrices of dimension larger than 1000 which makes it infeasible to use classical methods for the solution of the Riccati equations as these are of cubic complexity. In the late 90's Li and Penzl $[6,36]$ independently proposed a method for the efficient solution of large sparse Lyapunov equations. These methods are based on earlier work of Wachspress [43] on the application of an ADI-like method exploiting sparsity and the oftenly encountered very low numerical rank of their solutions.

The method developed by $\mathrm{Li}$ and Penzl can also be used to solve the large sparse Riccati equations appearing in this approach, since the Fréchet derivative of the Riccati operator is a Lyapunov operator. Thus we can apply Newton's method to the nonlinear matrix Riccati equations and in each step solve the Lyapunov equations efficiently by the ADI approach.

For the finite final time, it is shown in [7] that backward differentiation (BDF) methods can be combined with the above Newton-ADI-method to solve the differential Riccati equation (10) efficiently.

\section{Low Rank Cholesky Factor ADI Newton Method}

We will consider a system of the form (4), (5) here to characterize the low rank Cholesky factor ADI Newton method. It is sufficient to consider this case, because a finite-dimensional system of the form

$$
\begin{aligned}
M \dot{x}(t) & =A x(t)+B u(t) \\
y(t) & =C x(t) \\
x(0) & =x_{0}
\end{aligned}
$$

can easily be transformed into the representation (4), (5) by the following procedure. First split the matrix $M$ into $M=M_{L} M_{U}$ (where $M_{L}=M_{U}^{T}$ in the symmetric positive semidefinite case) and define $z(t):=M_{U} x(t)$. Then

$$
\dot{z}(t)=\dot{M}_{U} x(t)+M_{U} \dot{x}(t)=M_{U} \dot{x}(t)
$$

and by defining

$$
\begin{aligned}
\tilde{A} & :=M_{L}^{-1} A M_{U}^{-1}, \\
\tilde{B} & :=M_{L}^{-1} B, \\
\tilde{C} & :=C M_{U}^{-1},
\end{aligned}
$$

we can rewrite the system in the form (4), (5). The mass matrix from the finite element semidiscretization of the heat equation is always symmetric and positive definite and thus we can always apply the above procedure to the finite-dimensional systems. There also exists a method which avoids decompositions of $M$ by rewriting the linear systems of equation arising inside the ADI method instead of the control system (see [5] for details). We discussed the above method in detail here, because it is used in the LyaPack software package used to solve the Riccati equations in our implementation. 

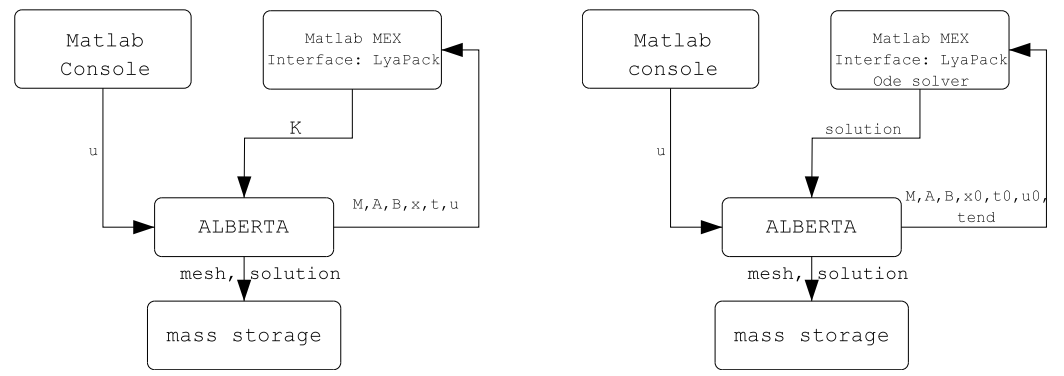

Fig. 2. Data flow in the horizontal (left) and vertical (right) method of lines implementations

The low rank Cholesky factor ADI Newton method implemented in LyaPack can be seen as a modification of the classical Newton-like method for algebraic Riccati equations (Kleinman iteration, [30]), where the Lyapunov subproblems in each Newton step are solved by the low rank Cholesky factor ADI method. The most important feature of this method is that it does not work with the generally full $n \times n$ iterates $X_{i}$ but with low rank Cholesky factors thereof $\left(Z_{i} Z_{i}^{T}=X_{i}\right)$. The rank of $Z_{i}$ is generally full but it has $r_{i} \ll n$ columns which drastically reduces memory consumption and computational complexity. LyaPack also provides functions iterating directly on the rectangular (number of rows $=$ number of system inputs $\ll n$ ) feedback matrix possibly reducing the complexity even further.

\subsection{Implementation Details}

For the implementation we combine the software packages LyaPack ${ }^{1}$ (see [37]) and ALBERTA $^{2}$ (see [39]). LyaPack is a collection of MATLAB-routines for solving large-scale Lyapunov equations, LQR problems, and model reduction tasks. Therefore we used Matlab to initialize the computation. That means we setup the initial control parameters and the time measurement routines at a MATLAB-console. After that the finite element discretization is generated by a mex call to a C-function utilizing the finite element method (fem) library ALBERTA. Inside this routine the system matrices $M, A$ and $B$ are assembled. After system assembly the program returns to the MATLAB prompt providing the matrices and problem data like current time and temperature profile. Now the matrices $C, Q$, and $R$ are initialized and LyaPack is used to compute the feedback matrix.

In case of the horizontal method of lines the program now returns to the ALBERTA subroutine providing the feedback and with it the new control parameters for the boundary conditions. With these it continues the standard

\footnotetext{
${ }^{1}$ available from: http://www.tu-chemnitz.de/

${ }^{2}$ available from: http://www.alberta-fem.de
} 
forward computation updating the boundary conditions by use of the feedback matrix.

In case of the vertical method of lines the feedback is used to generate the closed-loop system. This is then solved with a standard ODE-solver using MATLAB. After that the program uses the ALBERTA function again to store the solution on a mass storage device for visualization and post processing tasks in the same format used in the above case.

Both implementations have their advantages. The horizontal method of lines implementation can use operator information for the selection of (time) stepsizes and can easily be generalized to what might be called an adaptive LQR system, where the idea is to stabilize a system with nonlinear PDE constraints by systems for local (in time) linearizations of the constraints. This method is similar to receding horizon- or model predictive control techniques which will be addressed in Section 4. On the other hand the vertical method of lines implementation is easier to generalize to tracking-type control systems (see Section 2.2).

To close this section on the implementation details we give a table showing what our current implementation is capable of computing.

\begin{tabular}{|c|c|c|c|}
\hline & $x_{e x t, k}$ as control in (12) & $\frac{\kappa_{k}}{\lambda}$ as control in (12) & tracking \\
\hline vertical m.o.l. & $\mathrm{X}$ & + & $\mathrm{X}$ \\
\hline linear horiz. m.o.l. & $\mathrm{X}$ & + & - \\
\hline nonlinear horiz. m.o.l. & $\mathrm{O}$ & + & - \\
\hline
\end{tabular}

In the tabular an $\mathrm{X}$ denotes a fully supported feature. That means the feature is implemented and there exists a rigorous theory for this approach. An $\mathrm{O}$ denotes a feature which is fully implemented but the theoretical backing is not complete. The features marked ' + ' already give promising results although they are not covered by the theory. So under slight changes in the implementation (e.g. a posteriori error estimates) they might become fully valid and theoretically confirmed in future research.

\subsection{Numerical Results}

We will now present an example of a tracking control system.

We want to control the state (temperature distribution) to constant $700^{\circ} \mathrm{C}$. For the particular problem considered here one also knows the reference control which has to be applied to stay at this state. From (12) it is easy to see that we have to introduce an exterior temperature (cooling temperature) of $700^{\circ} \mathrm{C}$, because (12) then becomes an isolation boundary condition.

It is in general not necessary to know $\tilde{u}$ for this approach. We have seen in Section 2.2 that we only need to know that there exists such a control. We do not have to know the reference control itself for the computations, because to calculate the inhomogeneity $f$ we only use $\tilde{x}$ and its derivative with respect 

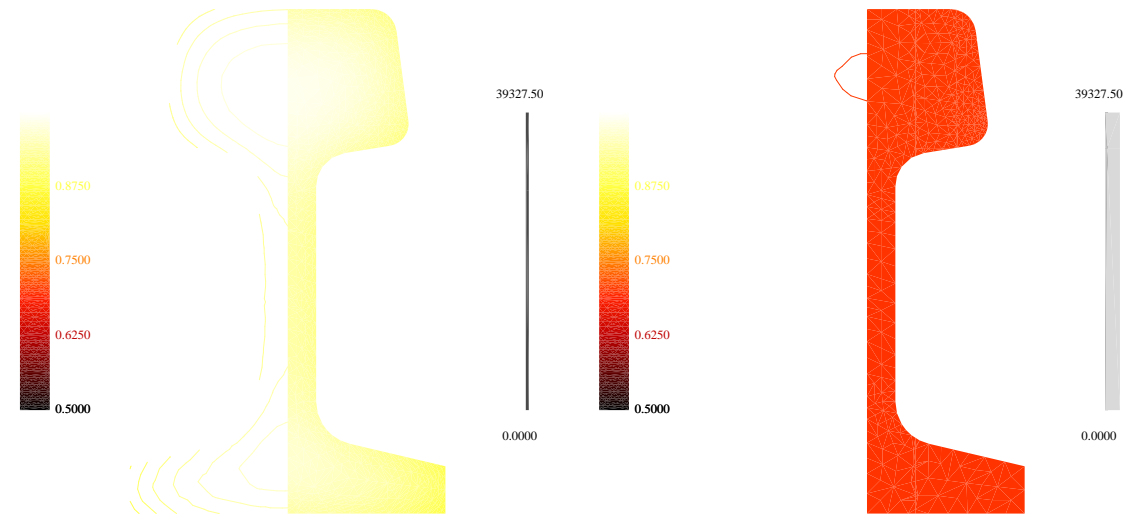

Fig. 3. Initial (left) and final (right) temperature distribution for a tracking type system steering to constant $700^{\circ} \mathrm{C}$.
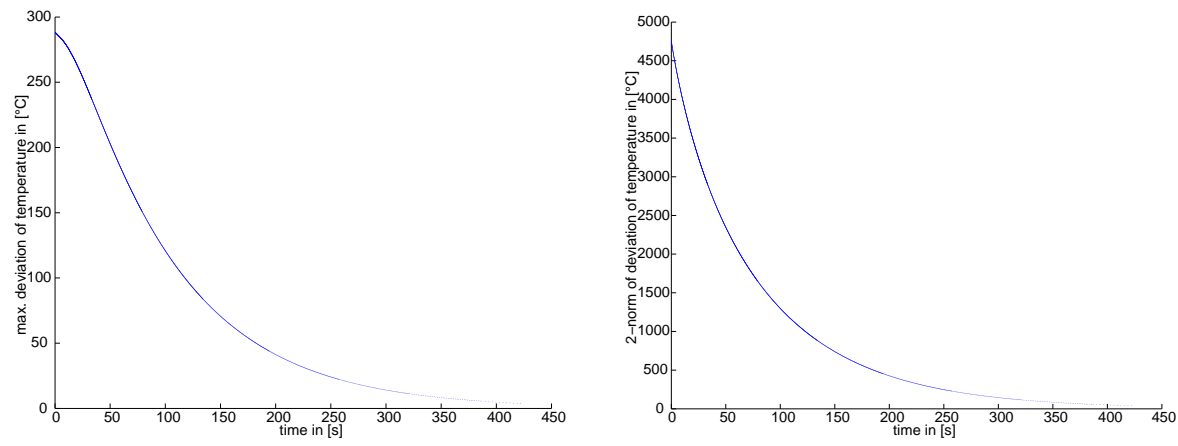

Fig. 4. Deviation of temperature from the reference $\left(700^{\circ} \mathrm{C}\right)$ in maximum norm (left) and Euclidian norm (right)

to time. On the other hand we might need $\tilde{u}$ to regain the real control $u$ from the artificial control $v$.

We start the calculation with the same initial temperature (see Figure 3 on the left) distribution we already used e.g. in [9]. The computational time horizon is equivalent to approximately 7 minutes of real time. The time-bars in Figure 3 have to be scaled down by a factor of 100 (see [9] for details on the scalings) to read real time in seconds. The temperatures are scaled such that 1.0 is equivalent to $1000^{\circ} \mathrm{C}$. The isolines in Figure 3 are plotted at a distance of $15^{\circ} \mathrm{C}$. Thus from Figure 3 we can conclude that the maximum deviation of temperatures from $700^{\circ} \mathrm{C}$ is smaller than $15^{\circ} \mathrm{C}$. In fact it is only $3.84^{\circ} \mathrm{C}$ after approximately 7 minutes. The average deviation at that time is already at about $0.186^{\circ} \mathrm{C}$ (also compare Figure 4).

Figure 5 shows the evolution of the control parameters (i.e., temperatures of the cooling fluid) on the right. The plotted values represent the real control 

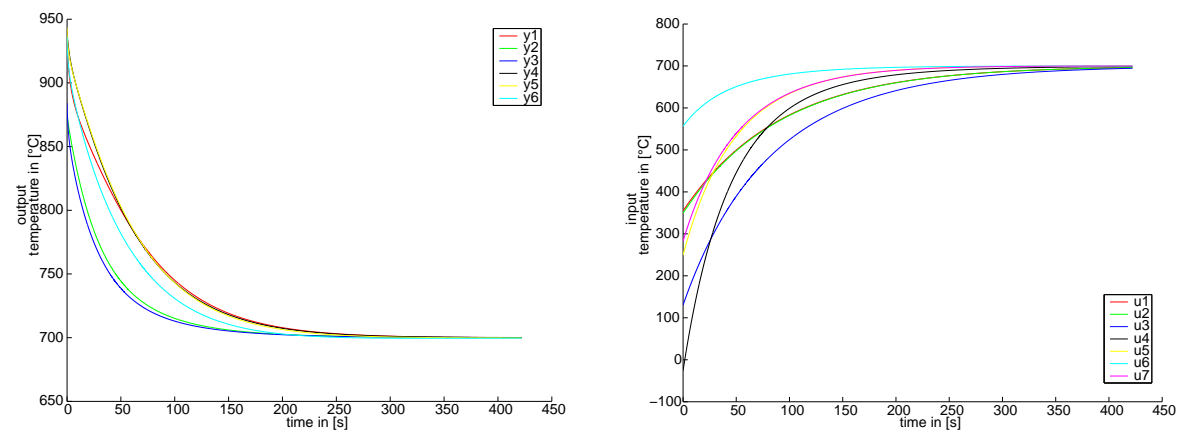

Fig. 5. Evolution of temperature at the outputs (left) and control inputs (right)

temperatures one would have to apply. In comparison to $v=u-\tilde{u}$ in (16) we already added the reference control $\tilde{u}$ of $700^{\circ} \mathrm{C}$ to the computed values of $v$.

\section{Nonlinear Parabolic Systems}

Nonlinear problems will arise if the system equation or the boundary conditions are nonlinear. In the following we consider the minimization problem

$$
\min _{\mathbf{u} \in L^{2}\left(0, T_{f} ; \mathcal{U}\right)} \int_{0}^{T_{f}} \mathbf{g}(\mathbf{y}(t), \mathbf{u}(t)) d t, \quad 0<T_{f} \leq \infty
$$

subject to the semilinear equation

$$
\dot{\mathbf{x}}=\mathbf{f}(\mathbf{x})+\mathbf{B u}(t), \quad t \in\left[0, T_{f}\right], \quad \mathbf{x}(0)=\mathbf{x}_{0} .
$$

The idea of receding horizon control (RHC) or model predictive control (MPC) is to decompose the time interval $\left[0, T_{f}\right]$ in (19) in smaller subintervals $\left[T_{i}, T_{i+1}\right]$ with

$$
0=T_{0}<T_{1}<T_{2}<\ldots<T_{\ell-1}<T_{\ell}=T_{f}
$$

and

$$
T \geq \max \left\{T_{i+1}-T_{i} \mid i=0,1, \ldots, \ell-1\right\}
$$

for given $T$. Now we have to solve optimal control problems on the time frames $\left[T_{i}, T_{i}+T\right]$ successively, that is we replace (18) and (19) by

$$
\min _{\mathbf{u} \in L^{2}\left(T_{i}, T_{i}+T ; \mathcal{U}\right)} \int_{T_{i}}^{T_{i}+T} \mathbf{g}(\mathbf{y}(t), \mathbf{u}(t)) d t+\mathbf{g}_{f}\left(\mathbf{x}\left(T_{i}+T\right)\right)
$$

subject to the dynamical semilinear system 


$$
\dot{\mathbf{x}}(t)=\mathbf{f}(\mathbf{x}(t))+\mathbf{B u}(t) \text { for } t \geq T_{i}, \quad \mathbf{u}(t) \in \mathcal{U},
$$

and the initial condition

$$
\mathbf{x}\left(T_{i}\right)=\mathbf{x}_{*}\left(T_{i}\right) .
$$

Here $\mathbf{u}_{*}$ is the optimal control and $\mathbf{x}_{*}$ the optimal trajectory for the optimal control problem on $\left[T_{i-1}, T_{i-1}+T\right]$. The second term $\mathbf{g}_{f}\left(\mathbf{x}\left(T_{i}+T\right)\right)$ in the cost functional (20) is called terminal cost and penalizes the states at the end of the finite horizon. It is required to establish the asymptotic stabilization property of the MPC scheme. To obtain the approximated optimal control on $\left[0, T_{f}\right]$ we have to compose the optimal controls on the subintervals $\left[T_{i}, T_{i+1}\right]$.

The strategy of MPC/RHC is used successfully in particular for control problems with ordinary differential equations, e.g. [1, 15]. The literature also provides research into partial differential equations, see [14, 16, 23, 24], where different techniques are used for solving the subproblems (20)-(22).

We want to present a tracking approach which was introduced in $[27,28$, 29]. In [29] the authors use a linear quadratic Gaussian (LQG) design which allows to include noise and observers into the model. Therefor we consider equation (19) as a nonlinear stochastic system

$$
\dot{\mathbf{x}}=\mathbf{f}(\mathbf{x})+\mathbf{B u}(t)+\mathbf{d}(t), \quad t \in\left[0, T_{f}\right], \quad \mathbf{x}(0)=\mathbf{x}_{0},
$$

where $\mathbf{d}(t)$ is an unknown Gaussian disturbance process. The observation process

$$
\mathbf{y}(t)=\mathbf{C x}(t)+\mathbf{n}(t)
$$

provides partial observations of the state $\mathbf{x}(t)$, where $\mathbf{n}(t)$ is a measurement noise process.

Now we consider the time frame $\left[T_{i}, T_{i}+T\right]$ and define an operating point, for example

$$
\overline{\mathbf{x}}=\frac{1}{T} \int_{T_{i}}^{T_{i}+T} \overline{\mathbf{x}}^{*}(t) d t \quad \text { or } \quad \overline{\mathbf{x}}=\overline{\mathbf{x}}^{*}\left(T_{i}+T\right)
$$

where $\left(\overline{\mathbf{u}}^{*}, \overline{\mathbf{x}}^{*}\right)$ is the reference pair on $\left[T_{i}, T_{i}+T\right]$ which is known from applications or results from an open-loop computation. If we linearize $\mathbf{f}(\mathbf{x})$ at $\overline{\mathbf{x}}$, we will obtain the following linear optimal control problem on $\left[T_{i}, T_{i}+T\right]$ :

$$
\min _{\tilde{\mathbf{u}} \in L^{2}\left(T_{i}, T_{i}+T ; \mathcal{U}\right)} \frac{1}{2} \int_{T_{i}}^{T_{i}+T}\langle\mathbf{z}, \mathbf{Q z z}\rangle_{\mathcal{Y}}+\langle\tilde{\mathbf{u}}, \mathbf{R} \tilde{\mathbf{u}}\rangle_{\mathcal{U}} d t+\mathbf{g}_{f}\left(\mathbf{x}\left(T_{i}+T\right)\right)
$$

subject to

$$
\begin{aligned}
\dot{\mathbf{z}}(t) & =\mathbf{A} \mathbf{z}(t)+\mathbf{B} \tilde{\mathbf{u}}(t)+\mathbf{d}(t), \quad \mathbf{z}(0)=\eta_{0}, \\
\tilde{\mathbf{y}}(t) & =\mathbf{C} \mathbf{z}(t)+\mathbf{n}(t)
\end{aligned}
$$

where 


$$
\mathbf{z}(t):=\mathbf{x}(t)-\overline{\mathbf{x}}^{*}(t), \quad \tilde{\mathbf{u}}(t):=\mathbf{u}(t)-\overline{\mathbf{u}}^{*}(t)
$$

and

$$
\mathbf{A}:=\frac{d}{d \mathbf{x}} \mathbf{f}(\overline{\mathbf{x}}) .
$$

It can be shown, see e.g. [15], that if the terminal cost $\mathbf{g}_{f}$ bounds the infinite horizon cost for the nonlinear system (starting from $T_{i}+T$ ), the cost function to be minimized is an upper bound for

$$
\min _{\tilde{\mathbf{u}} \in L^{2}\left(T_{i}, \infty ; \mathcal{U}\right)} \frac{1}{2} \int_{T_{i}}^{\infty}\langle\mathbf{z}, \mathbf{Q z z}\rangle_{\mathcal{Y}}+\langle\tilde{\mathbf{u}}, \mathbf{R} \tilde{\mathbf{u}}\rangle_{\mathcal{U}} d t
$$

So we can consider the infinite time case on every time frame. After application of the minimum principle we have to solve the algebraic Riccati equation (8), which corresponds to the LQR problem, as well as the dual equation

$$
\tilde{\Re}(\mathbf{W}):=\mathbf{A W}+\mathbf{W A}^{*}-\mathbf{W C}^{*} \mathbf{C W}+\mathbf{S}=0
$$

for the state estimation by using a Kalman filter, where $\mathbf{S}$ is an appropriate positive semidefinite operator. The best estimate $\hat{\mathbf{x}}$ can be obtained by solving the so called compensator equation

$$
\begin{aligned}
\dot{\hat{\mathbf{x}}}(t) & =\mathbf{A}(\overline{\mathbf{x}})\left(\hat{\mathbf{x}}(t)-\overline{\mathbf{x}}^{*}(t)\right)+\mathbf{f}\left(\overline{\mathbf{x}}^{*}(t)\right)+\mathbf{B u}(t)+\mathbf{W}_{*} \mathbf{C}^{*}(y(t)-\mathbf{C} \hat{\mathbf{x}}(t)), \\
\hat{\mathbf{x}}(0) & =\mathbf{x}_{0}+\eta_{0},
\end{aligned}
$$

where $\mathbf{W}_{*}$ is the positive semidefinite solution to (26). The associated feedback law is now given as

$$
\mathbf{u}(t)=\mathbf{u}^{*}(t)-\mathbf{R}^{-1} \mathbf{B}^{*} \mathbf{P}_{*}\left(\hat{\mathbf{x}}(t)-\overline{\mathbf{x}}^{*}(t)\right)
$$

where $\mathbf{P}_{*}$ is the positive semidefinite solution to (8).

Now we have computed the solution on the time frame $\left[T_{i}, T_{i+1}\right]$. In the next step we determine the solution on $\left[T_{i+1}, T_{i+2}\right]$ by repeating the procedure above, that is linearization, solving the two dual algebraic Riccati equations and determination of the optimal control. Since the current horizon is moving forward this strategy is called receding horizon control or moving horizon control.

The numerical implementation for such problems is similar to that described in Section 3. We need efficient algorithms to solve the two Riccati equations and the ordinary differential equation in every time frame. Details can be found in Sections 3.1 and 3.2.

It is also possible to linearize along a given (time-dependent) reference trajectory instead of using a constant operating point as in (24). Then we have to solve two differential Riccati equations which have the following form

$$
\begin{array}{r}
\dot{\mathbf{P}}(t)+\mathbf{A}(t)^{*} \mathbf{P}(t)+\mathbf{P}(t) \mathbf{A}(t)-\mathbf{P}(t) \mathbf{B R}^{-1} \mathbf{B}^{*} \mathbf{P}(t)+\mathbf{C}^{*} \mathbf{Q} \mathbf{C}=0 \\
\dot{\mathbf{W}}(t)+\mathbf{A}(t) \mathbf{W}(t)+\mathbf{W}(t) \mathbf{A}(t)^{*}-\mathbf{W}(t) \mathbf{C}^{*} \mathbf{C} \mathbf{W}(t)+\mathbf{S}=0
\end{array}
$$


where $\mathbf{A}(t) \equiv \mathbf{A}\left(\overline{\mathbf{x}}^{*}(t)\right)$. For the numerical solution of the differential Riccati equation we refer to [7].

The numerical implementation of this approach for nonlinear parabolictype problems such as semilinear and quasilinear heat, convection-diffusion, and reaction-diffusion equations is under current investigation. For solving the sub-problems on each time frame, we make intensive use of the algorithms developed for the linear case discussed in Section 3.

\section{Acknowledgments}

The authors wish to thank Hermann Mena from Escuela Politécnica Nacional Quito (Ecuador) for his contributions in the finite final time case.

\section{References}

1. F. Allgöwer, T.Badgwell, J. Qin, J. Rawlings, And S. Wright, Nonlinear predictive control and moving horizon estimation - an introductory overview, in Advances in Control, P. Frank, ed., Springer-Verlag, Berlin/Heidelberg, 1999, pp. 391-449.

2. A. Balakrishnan, Boundary control of parabolic equations: $L-Q-R$ theory., in Theory of nonlinear operators, Proc. 5th int. Summer Sch., no. 6N in Abh. Akad. Wiss. DDR 1978, Berlin, 1977, Akademie-Verlag, pp. 11-23.

3. H. BANKs, ed., Control and Estimation in Distributed Parameter Systems, vol. 11 of Frontiers in Applied Mathematics, SIAM, Philadelphia, PA, 1992.

4. H. BANKS AND K. KUnISCH, The linear regulator problem for parabolic systems, SIAM J. Cont. Optim., 22 (1984), pp. 684-698.

5. P. Benner, Solving large-scale control problems, IEEE Control Systems Magazine, 14 (2004), pp. 44-59.

6. P. Benner, J.-R. LI, AND T. PenzL, Numerical solution of large Lyapunov equations, Riccati equations, and linear-quadratic control problems. Unpublished manuscript, 2000.

7. P. Benner AND H. MENA, BDF methods for large-scale differential Riccati equations, in Proc. of Mathematical Theory of Network and Systems, MTNS 2004, B. D. Moor, B. Motmans, J. Willems, P. V. Dooren, and V. Blondel, eds., 2004.

8. P. BenNer AND J. SAAK, Efficient numerical solution of the LQR-problem for the heat equation, Proc. Appl. Math. Mech., 4 (2004), pp. 648-649.

9. — Linear-quadratic regulator design for optimal cooling of steel profiles, Tech. Rep. SFB393/05-05, Sonderforschungsbereich 393 Parallele Numerische Simulation für Physik und Kontinuumsmechanik, TU Chemnitz, D-09107 Chemnitz (Germany), 2005. Available from http://www.tu-chemnitz.de/sfb393/ sfb05pr.html.

10. — A semi-discretized heat transfer model for optimal cooling of steel profiles, in Dimension Reduction of Large-Scale Systems, P. Benner, V. Mehrmann, and D. Sorensen, eds., Lecture Notes in Computational Science and Engineering, Springer-Verlag, Berlin/Heidelberg, Germany, 2005, pp. 353-356. 
11. A. Bensoussan, G. D. Prato, M. Delfour, and S. Mitter, Representation and Control of Infinite Dimensional Systems, Volume I, Systems \& Control: Foundations \& Applications, Birkäuser, Boston, Basel, Berlin, 1992.

12. — Representation and Control of Infinite Dimensional Systems, Volume II, Systems \& Control: Foundations \& Applications, Birkäuser, Boston, Basel, Berlin, 1992

13. L. Biegler, O. Ghattas, M. Heinkenschloss and B. van Bloemen WaAnDERS, eds., Large-Scale PDE-Constrained Optimization, vol. 30 of Lecture Notes in Computational Science and Engineering, Springer-Verlag, Berlin, 2003.

14. M. Böhm, M. Demetriou, S. Reich, And I. Rosen, Model reference adaptive control of distributed parameter systems, SIAM J. Cont. Optim., 36 (1998), pp. 33-81.

15. C.-H. CHEn, A quasi-infinite horizon nonlinear model predictive control scheme with guaranteed stability, Automatica, 34 (1998), pp. 1205-1217.

16. H. ChOI, M. Hinze, AND K. KunisCH, Instantaneous control of backward-facing step flows, Appl. Numer. Math., 31 (1999), pp. 133-158.

17. R. Curtain And T. Pritchard, Infinite Dimensional Linear System Theory, vol. 8 of Lecture Notes in Control and Information Sciences, Springer-Verlag, New York, 1978.

18. R. Curtain And H. Zwart, An Introduction to Infinite-Dimensional Linear Systems Theory, vol. 21 of Texts in Applied Mathematics, Springer-Verlag, New York, 1995.

19. K. EPPler AND F. TRÖLtzsch, Discrete and continuous optimal control strategies in the selective cooling of steel profiles, Z. Angew. Math. Mech., 81 (2001), pp. 247-248.

20. - Discrete and continuous optimal control strategies in the selective cooling of steel profiles, Preprint 01-3, DFG Schwerpunktprogramm EchtzeitOptimierung groer Systeme, 2001. Available from http://www.zib.de/ dfg-echtzeit/Publikationen/Preprints/Preprint-01-3.html.

21. J. GIBSON, The Riccati integral equation for optimal control problems in Hilbert spaces, SIAM J. Cont. Optim., 17 (1979), pp. 537-565.

22. S. Godunov, Ordinary Differential Equations with Constant Coefficient., vol. 169 of Translations of Mathematical Monographs, American Mathematical Society, 1997.

23. M. Hinze, Optimal and instantaneous control of the instationary Navier-Stokes equations, Habilitationsschrift, TU Berlin, Fachbereich Mathematik, 2002.

24. M. Hinze And S. Volkwein, Analysis of instantaneous control for the Burgers equation, Optim. Methods Softw., 18 (2002), pp. 299-315.

25. K.-H. Hoffmann, I. Lasiecka, G. Leugering, J. Sprekels, And F. Tröltzsch, eds., Optimal control of complex structures. Proceedings of the international conference, Oberwolfach, Germany, June 4-10, 2000, vol. 139 of ISNM, International Series of Numerical Mathematics, Birkhäuser, Basel, Switzerland, 2002.

26. K.-H. Hoffmann, G. Leugering, and F. Tröltzsch, eds., Optimal control of partial differential equations. Proceedings of the IFIP WG 7.2 international conference, Chemnitz, Germany, April 20-25, 1998, vol. 133 of ISNM, International Series of Numerical Mathematics, Birkhäuser, Basel, Switzerland, 1999.

27. K. Ito AND K. Kunisch, On asymptotic properties of receding horizon optimal control, SIAM J. Cont. Optim., 40 (2001), pp. 1455-1472. 
28. — Receding horizon optimal control for infinite dimensional systems, ESAIM: Control Optim. Calc. Var., 8 (2002), pp. 741-760.

29. — Receding horizon control with incomplete observations. Preprint, Oct. 2003.

30. D. Kleinman, On an iterative technique for Riccati equation computations, IEEE Trans. Automat. Control, AC-13 (1968), pp. 114-115.

31. R. Krengel, R. Standke, F. Tröltzsch, and H. Wehage, Mathematisches Modell einer optimal gesteuerten Abkühlung von Profilstählen in Kühlstrecken, Preprint 98-6, Fakultät für Mathematik TU Chemnitz, November 1997.

32. I. Lasiecka And R. Triggiani, Differential and Algebraic Riccati Equations with Application to Boundary/Point Control Problems: Continuous Theory and Approximation Theory, no. 164 in Lecture Notes in Control and Information Sciences, Springer-Verlag, Berlin, 1991.

33. — Control Theory for Partial Differential Equations: Continuous and Approximation Theories I. Abstract Parabolic Systems, Cambridge University Press, Cambridge, UK, 2000.

34. — Control theory for partial differential equations: Continuous and approximation theories II. Abstract hyperbolic-like systems over a finite time horizon, in Encyclopedia of Mathematics and its Applications, vol. 75, Cambridge University Press, Cambridge, 2000, pp. 645-1067.

35. J. Lions, Optimal Control of Systems Governed by Partial Differential Equations, Springer-Verlag, Berlin, FRG, 1971.

36. T. PEnZL, A cyclic low rank Smith method for large sparse Lyapunov equations, SIAM J. Sci. Comput., 21 (2000), pp. 1401-1418.

37. —, LYAPACK Users Guide, Tech. Rep. SFB393/00-33, Sonderforschungsbereich 393 Numerische Simulation auf massiv parallelen Rechnern, TU Chemnitz, 09107 Chemnitz, FRG, 2000. Available from http://www.tu-chemnitz.de/sfb393/sfb00pr.html.

38. J. SAAK, Effiziente numerische Lösung eines Optimalsteuerungsproblems für die Abkühlung von Stahlprofilen, Diplomarbeit, Fachbereich 3/Mathematik und Informatik, Universität Bremen, D-28334 Bremen, Sept. 2003.

39. A. Schmidt And K. Siebert, Design of Adaptive Finite Element Software; The Finite Element Toolbox ALBERTA, vol. 42 of Lecture Notes in Computational Science and Engineering, Springer-Verlag, Berlin/Heidelberg, 2005.

40. E. Sontag, Mathematical Control Theory, Springer-Verlag, New York, NY, 2nd ed., 1998.

41. F. TRÖLtzSCH, Optimale Steuerung partieller Differentialgleichungen - Theorie, Verfahren und Anwendungen, Vieweg, Wiesbaden, 2005. In German.

42. F. TRÖLtzSCh AND A. Unger, Fast solution of optimal control problems in the selective cooling of steel, Z. Angew. Math. Mech., 81 (2001), pp. 447-456.

43. E. WACHSPRESS, Iterative solution of the Lyapunov matrix equation, Appl. Math. Letters, 107 (1988), pp. 87-90.

44. J. ZABCZYK, Remarks on the algebraic Riccati equation, Appl. Math. Optim., 2 (1976), pp. 251-258. 